\title{
Tribbles homolog 1 enhances cholesterol efflux from oxidized low-density lipoprotein-loaded THP-1 macrophages
}

\author{
YANHUA FU, YANG ZHAO and BIN HUANG \\ Department of Internal Medicine, VIP Ward, The Fifth Affiliated Hospital of Zhengzhou University, \\ Zhengzhou, Henan 450052, P.R. China
}

Received March 22, 2016; Accepted March 3, 2017

DOI: $10.3892 /$ etm.2017.4551

\begin{abstract}
Macrophage foam cell formation plays a pivotal role in the pathogenesis of atherosclerosis. The protein Tribbles homolog 1 (Trib1), a member of the Tribbles protein family, functions as an adaptor or scaffold protein. Recent studies have indicated its implication in lipoprotein metabolism. In the present study, the role of Tribl in macrophage foam cell formation was investigated. Oil red $\mathrm{O}$ staining was used to analyze intracellular lipid deposition, while the effects of Trib1 overexpression on cholesterol efflux were also examined. Furthermore, quantitative polymerase chain reaction and western blot analysis were performed to measure the expression levels of genes involved in cholesterol efflux. The results revealed that overexpression of Trib1 inhibited lipid accumulation in oxidized low density lipoprotein-treated THP-1 macrophages and facilitated macrophage cholesterol efflux to apolipoprotein A-I. Overexpression of Trib1 also upregulated the expression levels of ATP-binding cassette A1 (ABCA1), ABCG1, liver X receptor $\alpha(\mathrm{LXR} \alpha)$ and peroxisome proliferator-activated receptor $\gamma(\operatorname{PPAR} \gamma)$. In addition, silencing of LXR $\alpha$ or PPAR $\gamma$ via small interfering RNA transfection significantly reversed the Tribl-induced cholesterol efflux. In conclusion, Trib1 inhibits macrophage foam cell formation and enhances cholesterol efflux, which is associated with regulation of the PPAR $\gamma, \mathrm{LXR} \alpha, \mathrm{ABCA} 1$ and $\mathrm{ABCG} 1$ expression levels.
\end{abstract}

\section{Introduction}

Atherosclerosis is a chronic inflammatory disease, characterized by progressive accumulation of lipids and cellular and fibrous elements in the arterial wall $(1,2)$. Macrophages

Correspondence to: Dr Yanhua Fu, Department of Internal Medicine, VIP Ward, The Fifth Affiliated Hospital of Zhengzhou University, 3 Kangfuqian Street, Erqi, Zhengzhou, Henan 450052, P.R. China

E-mail: drfuyanhua@qq.com

Key words: atherosclerosis, cholesterol efflux, foam cells, therapeutic target are involved in various aspects of atherosclerosis $(3,4)$. In particular, macrophage-mediated uptake of modified low-density lipoprotein (LDL) and subsequent transformation into foam cells serve a critical role in the development of atherosclerosis (5). Lipid accumulation in macrophages leads to the activation of signaling pathways that involve activation of peroxisome proliferator-activated receptor- $\gamma(\operatorname{PPAR} \gamma)$ and liver $\mathrm{X}$ receptor $\alpha(\mathrm{LXR} \alpha)$, which are transcription factors controlling the macrophage cholesterol homeostasis (6). Activated PPAR $\gamma$ and $\mathrm{LXR} \alpha$ are synergistically implicated in the transactivation of several genes, such as the ATP-binding cassette transporter (ABCA1) and ABCG1, the products of which are involved in regulating the macrophage cholesterol efflux and triggering reverse cholesterol transport (7), which is part of a 'self-protection mechanism' for macrophages.

The protein Tribbles homolog 1 (Trib1), a member of the recently identified Tribbles protein family, is considered to function as an adaptor or scaffold protein (8). Burkhardt et al have reported that hepatic expression of Tribl regulates the plasma levels of LDL-cholesterol (LDL-C), very-LDL (VLDL), and triglyceride (TG) in mice (9). In addition, Satoh et al demonstrated that mice lacking Trib1 expression in hematopoietic cells developed hypertriglyceridemia and insulin resistance in response to a high-fat diet (10). These authors also suggested that Trib1 is critical for adipose tissue maintenance and inhibition of metabolic disorders by regulating the differentiation of tissue-resident M2-like macrophages (10). The aforementioned findings indicate that Trib1 serves an important role in lipoprotein metabolism. However, the functions of Trib1 in cholesterol efflux remain largely unknown.

In the present study, the THP-1 cell model was used to explore the role of Trib1 in the formation of macrophage foam cells during atherosclerosis. The effect of overexpression of Tribl on lipid accumulation and cholesterol efflux was investigated in macrophages, and associated molecular mechanisms were also explored.

\section{Materials and methods}

Cell culture and differentiation. THP-1 human monocytic cell line was purchased from the Shanghai Cell Institute of the Chinese Academy of Sciences (Shanghai, China). THP-1 cells were cultured in RPMI-1640 medium supplemented with $10 \%$ fetal bovine serum, penicillin $(100 \mathrm{U} / \mathrm{ml})$ and 
streptomycin $(100 \mu \mathrm{g} / \mathrm{ml})$ at $37^{\circ} \mathrm{C}$ in $5 \% \mathrm{CO}_{2}$. To differentiate into macrophages, THP-1 monocytes were exposed to $160 \mathrm{nM}$ phorbol-12-myristate-13-acetate (Sigma-Aldrich, St. Louis, MO, USA) for $72 \mathrm{~h}$. The differentiated macrophages were washed three times with phosphate-buffered saline (PBS) and incubated in fresh serum-free medium containing $50 \mu \mathrm{g} / \mathrm{ml}$ oxidized LDL (ox-LDL; Yiyuan Biotechnology Co., Ltd., Guangzhou, China) for 48 h.

Cell transfection. In order to investigate the effect of Trib1 overexpression in THP-1 macrophages, the cells were transfected with pCMV6-Entry-Trib1 (Trib1 overexpressing group) or pCMV6-Entry vector (empty vector group) using Lipofectamine ${ }^{\circledR} 2000$ (Invitrogen; Thermo Fisher Scientific, Inc., Waltham, MA, USA) according to the manufacturer's instructions. The pCMV6-Entry-Trib1 and empty vector were purchased from OriGene Technologies, Inc. (Rockville, MD, USA). Non-transfected THP-1 macrophages were used as a control. For small interfering RNA (siRNA)-mediated knockdown experiments, THP-1 macrophages were co-transfected with pCMV6-Entry-Trib1 and LXR $\alpha$ siRNA or PPAR $\gamma$ siRNA (Santa Cruz Biotechnology Inc., Santa Cruz, CA, USA). After $24 \mathrm{~h}$ of transfection, cells were incubated with ox-LDL $(50 \mu \mathrm{g} / \mathrm{ml})$ in serum-free medium for an additional $24 \mathrm{~h}$ before conducting the expression or functional studies.

Morphological examination. Non-transfected or transfected THP-1 macrophages were cultured in chamber slides in serum-free medium. After 24-h incubation, cells were washed three times in PBS and fixed with 5\% formalin solution for $30 \mathrm{~min}$. Next, the cells were stained with oil red O (Sigma-Aldrich) for $30 \mathrm{~min}$, and counterstained with hematoxylin for $5 \mathrm{~min}$. Finally, cells were analyzed by light microscopy (Axio Imager 2; Zeiss, Oberkochen, Germany). Five selected high-power fields at a magnification of $x 400$ were randomly selected for examination. Semi-quantitative analysis of oil red $\mathrm{O}$ positive staining was conducted by the ImageJ software (version 1.48, National Institutes of Health, Bethesda, MD, USA).

Cholesterol efflux. In order to investigate the cholesterol efflux, non-transfected or transfected THP-1 macrophages $\left(5 \times 10^{5}\right.$ cells/well) were seeded into 12 -well plates, labeled with $0.5 \mu \mathrm{Ci} / \mathrm{ml}\left[{ }^{3} \mathrm{H}\right]$-cholesterol (PerkinElmer, Waltham, MA, USA) in media containing $0.2 \%$ bovine serum albumin for $24 \mathrm{~h}$ and then washed with fresh media. Then, cells were washed with PBS and incubated in the presence of $10 \mu \mathrm{g} / \mathrm{ml}$ apolipoprotein A-I (apoA-I; EMD Millipore, Billerica, MA, USA) for $18 \mathrm{~h}$. Medium and cell-associated $\left[{ }^{3} \mathrm{H}\right]$ cholesterol were examined through liquid scintillation counting. The percentage of cholesterol efflux was calculated with the following equation: [Total media radioactivity/(total cellular radioactivity + total media radioactivity)] x $100 \%$.

Reverse transcription-quantitative polymerase chain reaction (RT-qPCR) analysis. Total RNA was isolated from THP-1 macrophages with TRIzol reagent (Invitrogen; Thermo Fisher Scientific, Inc.). Total RNA concentration was measured by spectrophotometry with a NanoDrop 2000 Spectrophotometer (Thermo Fisher Scientific, Inc.). cDNA synthesis was performed using the PrimeScript RT reagent Kit according to the manufacturer's instructions (catalog no. RR037A; Takara, Shiga, Japan). qPCR analysis was then conducted using a Light Cycler-FastStart DNA Master SYBR-Green I kit (Roche Molecular Biochemicals, Manheim, Germany). The sequences of the primers used in the current study are shown in Table I. Data were analyzed with the $2^{-\Delta \Delta C q}$ relative quantification method (11). Values are presented as fold change relative to control cells.

Western blot analysis. After the indicated treatment, cells were washed with ice-cold PBS and harvested in lysis buffer [30 mM HEPES, pH 7.6, $30 \mathrm{mM} \mathrm{NaCl}, 1 \%$ Nonidet P-40 (vol/vol), $10 \%$ glycerol (vol/vol), $50 \mathrm{mM} \mathrm{NaF}$ and $10 \mathrm{mM} \mathrm{Na}$ pyrophosphate] supplemented with $5 \mathrm{mM}$ Na orthovanadate and protease inhibitors (Roche Diagnostics, Indianapolis, IN, USA). Cell lysates were collected by centrifugation at $14,000 \mathrm{x} \mathrm{g}$ for $5 \mathrm{~min}$ at $4^{\circ} \mathrm{C}$, and the protein concentration in total lysates was analyzed by the BCA protein assay kit (Pierce; Thermo Fisher Scientific, Inc.). Next, $20 \mu \mathrm{g}$ total protein were subjected to $10 \%$ SDS-PAGE and transferred onto a nitrocellulose membrane (Whatman; Sigma-Aldrich). Membranes were blocked overnight with 5\% non-fat dry milk in Tris-buffered saline containing $0.1 \%$ Tween- 20 for $1 \mathrm{~h}$ at room temperature. The membranes were then probed overnight at $4^{\circ} \mathrm{C}$ with the following primary antibodies: Anti-TRIB1 (ab137717; 1:200), anti-ABCA1 (ab18180; 1:200), anti-ABCG1 (ab155918; 1:200), anti-LXR $\alpha$ (ab82774; 1:200), anti-PPAR $\gamma($ ab24509; 1:200) and anti- $\beta$-actin (ab97379; 1:500; all from Abcam, Cambridge, MA, USA). Subsequently, samples were incubated with horseradish peroxidase-conjugated goat anti-mouse IgG (ab97265; 1:2,000 dilution) or goat anti-rabbit IgG (ab97200; 1:2,000 dilution; both from Abcam) for $1 \mathrm{~h}$ at room temperature. Final detection was performed using an ECL chemiluminescence system (Bio-Rad Laboratories, Inc., Hercules, CA, USA), and quantitative results were obtained using Quantity One software (version 4.4.0; Bio-Rad Laboratories, Inc.).

Statistical analysis. All data are expressed as the mean \pm standard deviation. Results were analyzed by one-way analysis of variance, followed by the Tukey's post hoc test, using SPSS version 11.0 software (SPSS, Inc., Chicago, IL, USA). P-values of $<0.05$ were considered to indicate differences that were statistically significant.

\section{Results}

Overexpression of Tribl inhibits lipid accumulation and enhances cholesterol efflux in ox-LDL-stimulated THP-1 macrophages. Transfection of THP-1 macrophages with pCMV6-Entry-Trib1 resulted in significant overexpression of Trib1 (increased by $\sim 8.6$-fold), as determined by western blot analysis $(\mathrm{P}<0.05$ vs. vector-transfected cells; Fig. 1A). Subsequently, in order to explore the intracellular lipid deposition in response to Trib1 overexpression in THP-1 macrophages, the intracellular cholesterol levels were analyzed. Forced expression of Trib1 significantly inhibited lipid accumulation as evidenced by oil red $\mathrm{O}$ staining $(\mathrm{P}<0.05$; Fig. 1B and C). Furthermore, exogenous expression of Trib1 significantly enhanced the apoA-I-mediated cholesterol efflux 
Table I. Primers used in quantitative real-time polymerase chain reaction analysis.

\begin{tabular}{lll}
\hline Gene & \multicolumn{1}{c}{ Forward primer } & \multicolumn{1}{c}{ Reverse primer } \\
\hline ABCA1 & 5'-ATCTCATAGTATGGAAGAATGTGAAGCT-3' & 5'-CGTACAACTATTGTATAACCATCTCCAAA-3' \\
ABCG1 & 5'-AGGTCTCAGCCTTCTAAAGTTCCTC-3' & 5'-TCTCTCGAAGTGAATGAAATTTATCG-3' \\
LXR $\alpha$ & 5'-TCAGCATCTTCTCTGCAGACCGG-3' & 5'-TCATTAGCATCCGTGGGAACA-3' \\
PPAR $\gamma$ & 5'-TGAACAAAGACGGGATG-3' & 5'-TCAAACTTGGGTTCCATGAT-3' \\
$\beta$-actin & 5'-TCATGAAGTGTGACGTTGACATCCGT-3' & 5'-CTTAGAAGCATTTGCGGTGCACGATG-3'
\end{tabular}

ABC, ATP-binding cassette transporter; LXR $\alpha$, liver X receptor $\alpha$; PPAR $\gamma$, peroxisome proliferator-activated receptor $\gamma$.

A

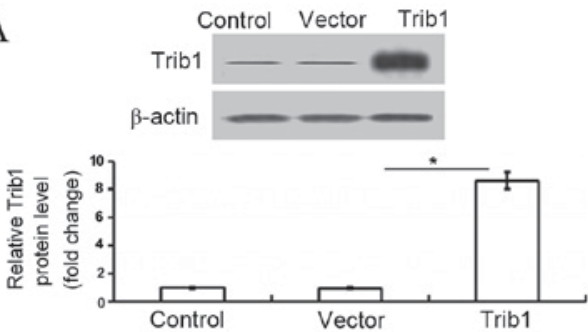

$\mathrm{C}$

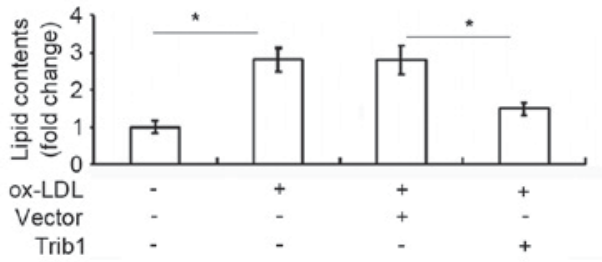

B

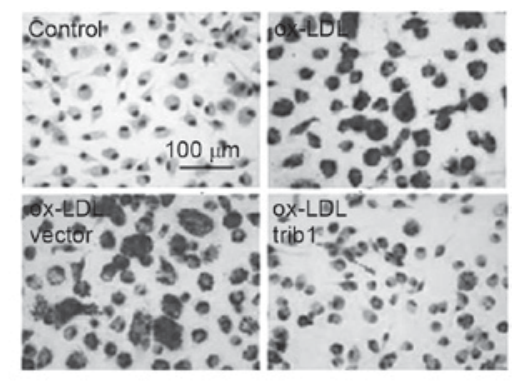

$\mathrm{D}$

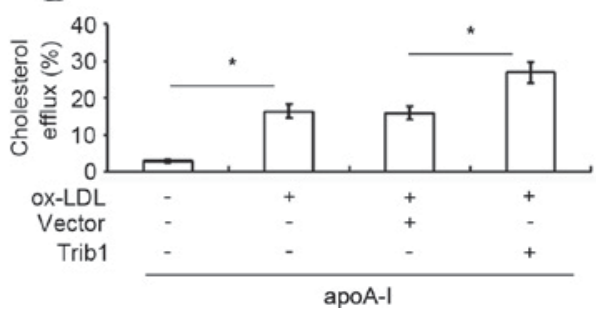

Figure 1. Effects of Trib1 on intracellular lipid accumulation and cholesterol efflux in THP-1 macrophages. Cells were transfected with pCMV6-Entry-Trib1 (Trib1 group) or pCMV6-Entry (Vector group) for $24 \mathrm{~h}$ and then incubated with $50 \mu \mathrm{g} / \mathrm{ml}$ ox-LDL in serum-free medium for $24 \mathrm{~h}$. (A) Expression of Trib1 protein, as determined by western blot analysis. (B and C) Lipid accumulation in cells was investigated by oil red O staining (magnification, x100). (D) Intracellular apolipoprotein A-I-mediated cholesterol efflux. Data are representative of the mean \pm standard deviation of duplicate wells from three independent experiments. " $\mathrm{P}<0.05$. Trib1, Tribbles homolog 1; ox-LDL, oxidized low-density lipoprotein.

in ox-LDL-stimulated THP-1 cells ( $\mathrm{P}<0.05$; Fig. 1D). These results revealed that Trib1 inhibited the intracellular lipid deposition due to increased apoA-I-mediated cholesterol efflux in THP-1 macrophages.

Forced expression of Tribl increases the expression of $A B C A 1, A B C G 1, L X R \alpha$ and PPAR $\gamma$. RT-qPCR analysis demonstrated that overexpression of Trib1 significantly increased the ABCA1, ABCG1, LXR $\alpha$ and PPAR $\gamma$ mRNA expression levels in ox-LDL-stimulated THP-1 macrophages $(\mathrm{P}<0.05$; Fig. 2A). Similarly, western blot analysis indicated that Trib1 overexpression led to a significant (1.5-2-fold) increase in the levels of ABCA1, ABCG1, LXR $\alpha$, and PPAR $\gamma$ protein $(\mathrm{P}<0.05$; Fig. $2 \mathrm{~B})$.

LXR o or PPAR $\gamma$ siRNA transfection attenuates Tribl-induced cholesterol efflux. Silencing of LXR $\alpha$ by siRNA transfection significantly decreased Tribl-induced cholesterol efflux in THP-1 macrophages transfected with pCMV6-Entry-Trib1, when compared with the cholesterol efflux in THP-1 macrophages-transfected with pCMV6-Entry-Trib1 and a control siRNA $(\mathrm{P}<0.05$; Fig. 3A). Furthermore, knockdown of
PPAR $\gamma$ by siRNA transfection also significantly attenuated the Tribl-induced cholesterol efflux compared with transfection with a control siRNA $(\mathrm{P}<0.05$; Fig. 3B).

\section{Discussion}

Genome-wide association studies identified that the genetic locus at human chromosome 8q24 has minor alleles associated with lower levels of plasma TG and LDL-C, as well as higher levels of high density lipoprotein-cholesterol $(12,13)$. This locus contains only one annotated gene, namely Trib1 (12,13). Burkhardt et al demonstrated that Trib1 is a regulator of lipoprotein metabolism in mice (9), and that hepatic-specific overexpression of Trib1 reduces the plasma TG and cholesterol levels by reducing VLDL production (9). Conversely, Trib1-knockout mice exhibited elevated levels of plasma TG and cholesterol due to increased VLDL production (9). In addition, Satoh et al suggested that Trib1 is critical for adipose tissue maintenance and suppression of metabolic disorders by controlling the differentiation of tissue-resident M2-like macrophages (10). These results indicate that Trib1 is implicated in the regulation of lipid metabolism. However, 

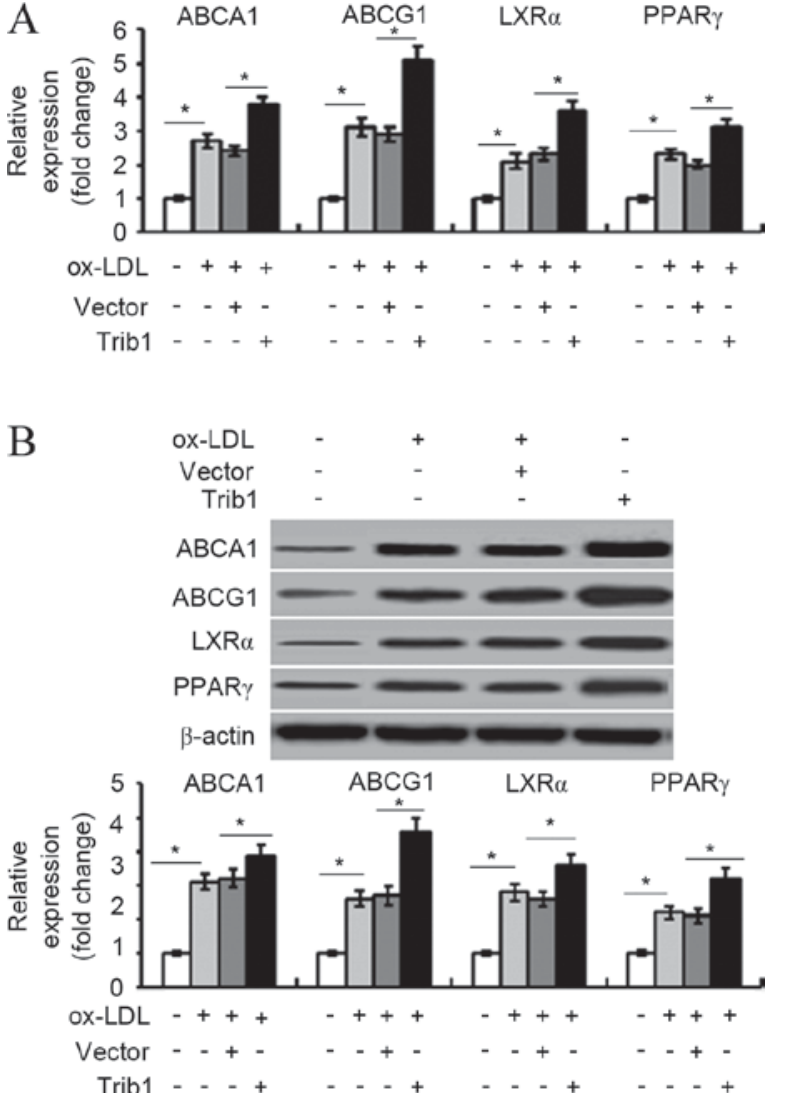

Figure 2. Effects of Trib1 on the (A) mRNA and (B) protein expression levels of ABCA1, ABCG1, LXR $\alpha$ and PPAR $\gamma$ in THP-1 macrophages, determined by quantitative polymerase chain reaction and western blot analysis, respectively. THP-1 macrophages were transfected with pCMV6-Entry-Trib1 or pCMV6-Entry for $24 \mathrm{~h}$ and then incubated with $50 \mu \mathrm{g} / \mathrm{ml}$ ox-LDL in serum-free medium for a further $24 \mathrm{~h}$. $\beta$-actin served as an internal control Data are presented as the mean \pm standard deviation of duplicate wells from three experiments. ${ }^{*} \mathrm{P}<0.05$. Trib1, Tribbles homolog 1; ox-LDL, oxidized low-density lipoprotein; ABC, ATP-binding cassette transporter; LXR $\alpha$, liver X receptor $\alpha$; PPAR $\gamma$, peroxisome proliferator-activated receptor $\gamma$.

the underlying mechanism throughout which Trib1 regulates lipid metabolism at the molecular level remains unclear. In the present study, the effect of Tribl overexpression on lipid accumulation and intracellular cholesterol efflux was investigated in ox-LDL-stimulated THP-1 macrophages. Transiently transfected THP-1 macrophages were used to examine the role of Trib1 in cholesterol homeostasis. The present results indicated that forced expression of Trib1 decreased intracellular lipid accumulation and enhanced cholesterol efflux in ox-LDL-exposed THP-1 macrophages.

PPAR $\gamma$ is a nuclear receptor that regulates immunity and inflammation $(14,15)$. Upon binding with its ligands, PPAR $\gamma$ activates and promotes cholesterol efflux from macrophages through the PPAR $\gamma$-LXR $\alpha$-ABCA1 signaling pathway (16). $\mathrm{LXR} \alpha$ is a nuclear receptor transcription factor (17) that when activated binds to the LXR response element in the promoter region of the LXR target genes, such as ABCA1 and $A B C G 1$, in order to regulate the expression of such genes (18). The current study examined the impact of overexpression of Trib1 on the expression of genes involved in macrophage cholesterol efflux. After transient transfection with pCMV6-Entry-Trib1, cellular responses were analyzed. Forced expression of Trib1 was found to induce the expression
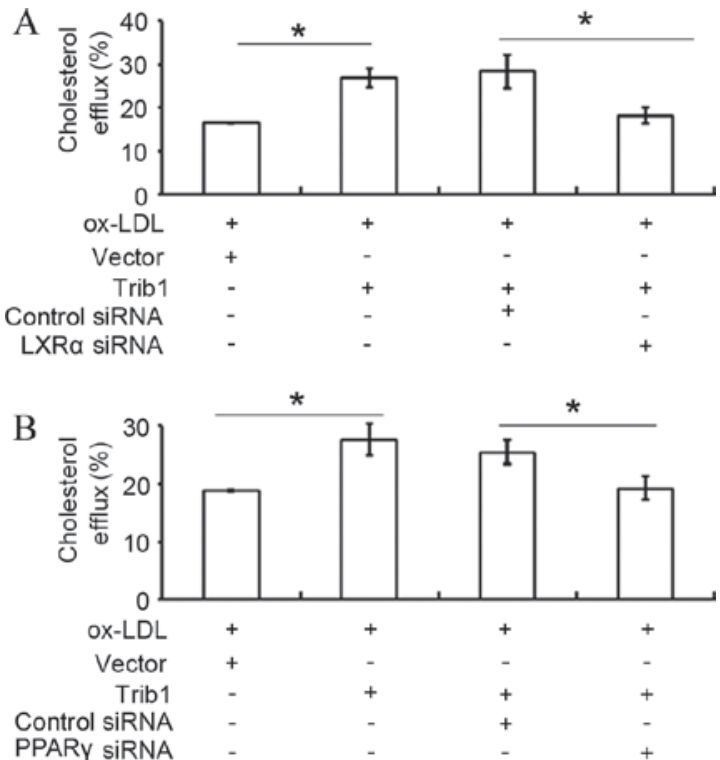

Figure 3. Effects of overexpression of Trib1 on apoA-I-mediated cholesterol efflux in LXR $\alpha$-silenced or PPAR $\gamma$-silenced and ox-LDL-stimulated THP-1 macrophages. Cells were co-transfected with pCMV6-Entry-Trib1 and (A) LXR $\alpha$ siRNA or (B) PPAR $\gamma$ siRNA for $24 \mathrm{~h}$, incubated with ox-LDL in serum-free medium for a further $24 \mathrm{~h}$ and then the intracellular apolipoprotein A-I-mediated cholesterol efflux was determined. Data are presented as the mean \pm standard deviation of duplicate wells from three experiments. ${ }^{*} \mathrm{P}<0.05$. Trib1, Tribbles homolog 1; ox-LDL, oxidized low-density lipoprotein; LXR $\alpha$, liver X receptor $\alpha$; PPAR $\gamma$, peroxisome proliferator-activated receptor $\gamma$.

of ABCA1, ABCG1, LXR $\alpha$ and PPAR $\gamma$ at the mRNA and protein levels. Furthermore, silencing of PPAR $\gamma$ or targeting LXR $\alpha$ by siRNA attenuated the Trib1-induced cholesterol efflux, indicated that Trib1-mediated cholesterol efflux may occur through the PPAR $\gamma$-LXR $\alpha$-ABCA1/ABCG1 signaling pathway. However, it is unclear how Trib1 regulates the PPAR $\gamma-L X R \alpha-A B C A 1 / A B C G 1$ signaling pathway. It has been suggested that CD36 activates PPAR $\gamma$ through the extracellular signal-regulated kinase (ERK)1/2-dependent cyclooxygenase 2 expression in macrophages, thereby promoting cholesterol and phospholipid efflux from macrophages (19). Similarly, sesamin has been demonstrated to improve macrophage cholesterol efflux through the PPAR $\gamma 1-\mathrm{LXR} \alpha$ pathway, which is dependent on mitogen-activated protein kinase (MAPK) signaling (20). It appears that MAPK signaling serves an important role in cholesterol efflux. Overexpression of Trib1 enhances ERK phosphorylation, reducing the apoptosis of leukemic cells upon interleukin-3 depletion and may be a key mediator between the RTK-MAPK pathway and the C/EBP transcription factor in myeloid leukemogenesis (21). In the present study, it was hypothesized that Trib1-mediated cholesterol efflux may occur through the MAPK signaling pathway; however, further experiments are required to test this hypothesis.

Although Tribl overexpression leads to enhanced cholesterol efflux from ox-LDL-loaded macrophages, it is still unclear whether Trib1 is necessary for cholesterol efflux. The effects of silencing Tribl on cholesterol efflux should be investigated further.

In conclusion, the results of the present study indicate that Trib1 inhibits lipid accumulation and enhances cholesterol efflux in ox-LDL-exposed THP-1 macrophages through the 
PPAR $\gamma$-LXR $\alpha$-ABCA1/ABCG1 signaling pathway. These data provide a rationale for investigating the potential of delivering Trib1 in the prevention of macrophage foam cell formation and atherosclerosis.

\section{References}

1. Moss JW, Davies TS, Garaiova I, Plummer SF, Michael DR and Ramji DP: A Unique combination of nutritionally active ingredients can prevent several key processes associated with atherosclerosis in vitro. PLoS One 11: e0151057, 2016.

2. Aluganti Narasimhulu C, Fernandez-Ruiz I, Selvarajan K, Jiang X, Sengupta B, Riad A and Parthasarathy S: Atherosclerosis-do we know enough already to prevent it? Curr Opin Pharmacol 27: 92-102, 2016

3. Conti P and Shaik-Dasthagirisaeb Y: Atherosclerosis: A chronic inflammatory disease mediated by mast cells. Cent Eur J Immunol 40: 380-386, 2015.

4. Rojas J, Salazar J, Martínez MS, Palmar J, Bautista J, Chávez-Castillo M, Gómez A and Bermúdez V: Macrophage heterogeneity and plasticity: Impact of macrophage biomarkers on atherosclerosis. Scientifica (Cairo) 2015: 851252, 2015.

5. Glass CK and Witztum JL: Atherosclerosis: The road ahead. Cell 104: 503-516, 2001.

6. Bogachev O, Majdalawieh A, Pan X, Zhang L and Ro HS: Adipocyte enhancer-binding protein 1 (AEBP1) (a novel macrophage proinflammatory mediator) overexpression promotes and ablation attenuates atherosclerosis in ApoE (-/-) and LDLR (-/-) mice. Mol Med 17: 1056-1064, 2011.

7. Zhou Q, Mei Y, Shoji T, Han X, Kaminski K, Oh GT, Ongusaha PP, Zhang K, Schmitt $\mathrm{H}$, Moser M, et al: Rho-associated coiled-coil-containing kinase 2 deficiency in bone marrow-derived cells leads to increased cholesterol efflux and decreased atherosclerosis. Circulation 126: 2236-2247, 2012.

8. Kiss-Toth E, Bagstaff SM, Sung HY, Jozsa V, Dempsey C, Caunt JC, Oxley KM, Wyllie DH, Polgar T, Harte M, et al: Human tribbles, a protein family controlling mitogen-activated protein kinase cascades. J Biol Chem 279: 42703-42708, 2004

9. Burkhardt R, Toh SA, Lagor WR, Birkeland A, Levin M, Li X, Robblee M, Fedorov VD, Yamamoto M, Satoh T, et al: Trib1 is a lipid- and myocardial infarction-associated gene that regulates hepatic lipogenesis and VLDL production in mice. J Clin Invest 120: 4410-4414, 2010.
10. Satoh T, Kidoya H, Naito H, Yamamoto M, Takemura N, Nakagawa K, Yoshioka Y, Morii E, Takakura N, Takeuchi O and Akira S: Critical role of Trib1 in differentiation of tissue-resident M2-like macrophages. Nature 495: 524-528, 2013.

11. Livak KJ and Schmittgen TD: Analysis of relative gene expression data using real-time quantitative PCR and the 2(-Delta Delta C(T)) Method. Methods 25: 402-408, 2001.

12. Kathiresan S, Melander O, Guiducci C, Surti A, Burtt NP Rieder MJ, Cooper GM, Roos C, Voight BF, Havulinna AS, et al: Six new loci associated with blood low-density lipoprotein cholesterol, high-density lipoprotein cholesterol or triglycerides in humans. Nat Genet 40: 189-197, 2008.

13. Willer CJ, Sanna S, Jackson AU, Scuteri A, Bonnycastle LL, Clarke R, Heath SC, Timpson NJ, Najjar SS, Stringham HM, et al: Newly identified loci that influence lipid concentrations and risk of coronary artery disease. Nat Genet 40: 161-169, 2008.

14. Glass CK and Saijo K: Nuclear receptor transrepression pathways that regulate inflammation in macrophages and T cells. Nat Rev Immunol 10: 365-376, 2010.

15. Villacorta L, Schopfer FJ, Zhang J, Freeman BA and Chen YE: PPARgamma and its ligands: Therapeutic implications in cardiovascular disease. Clin Sci (Lond) 116: 205-218, 2009.

16. Bouhlel MA, Staels B and Chinetti-Gbaguidi G: Peroxisome proliferator-activated receptors-from active regulators of macrophage biology to pharmacological targets in the treatment of cardiovascular disease. J Intern Med 263: 28-42, 2008.

17. Huang C: Natural modulators of liver X receptors. J Integr Med 12: 76-85, 2014.

18. Zelcer $\mathrm{N}$ and Tontonoz P: Liver X receptors as integrators of metabolic and inflammatory signaling. J Clin Invest 116: 607-614, 2006.

19. Bujold K, Rhainds D, Jossart C, Febbraio M, Marleau S and Ong H: CD36-mediated cholesterol efflux is associated with PPARgamma activation via a MAPK-dependent COX-2 pathway in macrophages. Cardiovasc Res 83: 457-464, 2009.

20. Majdalawieh AF and Ro HS: The anti-atherogenic properties of sesamin are mediated via improved macrophage cholesterol efflux through PPAR $\gamma 1-\mathrm{LXR} \alpha$ and MAPK signaling. Int J Vitam Nutr Res 84: 79-91, 2014.

21. Yokoyama T, Kanno Y, Yamazaki Y, Takahara T, Miyata S and Nakamura T: Trib1 links the MEK1/ERK pathway in myeloid leukemogenesis. Blood 116: 2768-2775, 2010. 